\title{
Intoxication volontaire par une tisane de feuilles de Datura**
}

\section{Self intoxication with a Datura leaves herbal tea**}

\section{Sabine COHEN ${ }^{(1) *}$, Claudette BERNY(1), Sylvie MEYRAN ${ }^{(2)}$, Anne MIALON ${ }^{(1)}$, Monique MANCHON ${ }^{(1)}$}
(1) Laboratoire des Urgences Biochimiques et Toxicologiques, CHLS, chemin du Grand Revoyet 69495 PIERRE-BENITE CEDEX
(2) Services des Urgences, Hôpital St Joseph - 69007 LYON

* Auteur à qui adresser la correspondance : Sabine COHEN, Laboratoire des Urgences Biochimiques et Toxicologiques, CHLS, chemin du Grand Revoyet - 69495 PIERRE-BENITE CEDEX Tél : 0478861237 - Fax : 0478862198 - E-mail : sabine.cohen@chu-lyon.fr

** Ce travail a été présenté au XI ${ }^{\mathrm{eme}}$ congrès de la Société Française de Toxicologie Analytique, 11-13 juin 2003, DINARD - FRANCE

(Reçu le 20 octobre 2003 ; accepté le 5 janvier 2004)

\section{RÉSUMÉ}

Le Datura appartient à la famille des Solanacées, plante contenant trois alcaloüdes toxiques : atropine, scopolamine et hyoscine. Utilisé en toxicomanie pour ses propriétés hallucinogènes, il peut être responsable d'intoxication volontaire. Toute la plante est toxique et son ingestion entraîne l'apparition d'un syndrome anticholinergique. Nous rapportons ici un cas d'intoxication volontaire dont le tableau clinique est celui d'une intoxication atropinique (agitation, hallucination, mydriase, tachycardie, rétention urinaire). Le traitement a été symptomatique et l'évolution favorable. Les analyses chromatographiques (Remedi ${ }^{\circledR}$ ) des urines $d u$ patient ainsi que d'une décoction retrouvée en sa possession ont permis de confirmer l'hypothèse d'une intoxication aiguë au Datura.

\section{MOTS-CLÉS}

Datura, intoxication, anticholinergique, CLHP.

\section{SUMMARY}

The Datura belongs to the family of Solanaceae. It contains three main toxic alkaloids : atropine, scopolamine and hyoscine. Used in toxicomania for its hallucinogenics properties, it can be the cause of voluntary intoxication. All the plant is toxic and consumption of any part can result in severe anticholinergic toxicity. We are reporting here one case of voluntary intoxication; symptoms are comparable to an atropine intoxication (agitation, hallucination, mydriasis, tachycardia, urine retention), traitement was symptomatic and evolution was favourable. The chromatographic analysis (Remedi ${ }^{\circledR}$ ) of patient's urines and of a decoction (found near the patient) confirm an acute intoxication to the Datura.

\section{KEY-WORDS}

Datura, intoxication, anticholinergic, HPLC. 


\section{Introduction}

Le Datura est une plante de la famille des Solanacées connue depuis longtemps pour ses propriétés pharmacologiques, toxiques et hallucinogènes. Utilisée comme plante ornementale pour ses grandes fleurs mauves ou blanches en forme de cloche, elle est rencontrée dans de nombreuses régions de France. Plusieurs espèces sont cultivées, la plus fréquente en France est Datura stramonium, également la plus toxique. La plante qui fleurit surtout l'été et l'automne peut produire de 500 à 5000 graines. Ce sont les graines qui sont les plus riches en alcaloïdes, mais toute la plante est toxique. A des fins récréatives ou toxicomaniaques, le Datura peut donc être consommé de différentes façons : infusion des feuilles ou des bogues, consommation des graines, ou inhalation en association à du tabac.

Plusieurs sites TREND (tendances récentes et nouvelles drogues) rapportent une augmentation de la consommation du Datura ; différentes hypothèses ont été émises pour expliquer ce phénomène : le faible coût de cette drogue et son accès facile, l'engouement actuel pour les produits d'origine naturelle. Le Datura semble être le plus souvent offert ou troqué et lorsqu'il est vendu son coût varie de 5 à 10 euros selon le type de préparation (1).

Nous rapportons ici le cas d'un patient intoxiqué admis au service des Urgences de l'Hôpital Saint-Joseph (Lyon).

\section{Observation}

Monsieur X, âgé de 25 ans est retrouvé inconscient sur la voie publique et adressé par les pompiers au Service des Urgences de l'hôpital Saint Joseph. Une bouteille contenant une boisson verdâtre est retrouvée en sa possession.

Dans le service le patient alterne entre des phases de somnolence et des phases d'agitation extrême nécessitant une contention physique. A l'entrée le score de Glasgow est à 15 . L'examen clinique montre un épistaxis, un hématome péri orbitaire, une mydriase bilatérale aréactive, un abdomen indolore et souple. Le patient présente des hallucinations et des douleurs crâniennes. Il ne présente aucun signe de focalisation neurologique, pas de déficit moteur ; le signe de Babinski est négatif, les réflexes ostéo-tendineux ne sont pas retrouvés. La température corporelle initiale est normale $\left(37,5^{\circ} \mathrm{C}\right)$.

Au niveau cardiovasculaire on observe une tachycardie régulière (130 battements par minute), la pression artérielle systolique (PAS) est normale $(120 \mathrm{~mm} \mathrm{Hg}$ ) mais la pression artérielle diastolique (PAD) est très faible
$(40 \mathrm{~mm} \mathrm{Hg})$. Une heure et demi après l'admission, la PAS augmente légèrement ( $149 \mathrm{~mm} \mathrm{Hg})$ et la PAD se normalise $(71 \mathrm{~mm} \mathrm{Hg})$ et quatre heures après, la tension artérielle est tout à fait normale.

L'électrocardiogramme (en dehors de la tachycardie) et les radiographies des poumons sont normaux. Quelques heures après l'admission, une sonde urinaire est mise en place en raison d'un globe vésical.

L'interrogatoire du patient oriente vers une prise possible d'acide, ecstasy, héroïne, cannabis ou d'absinthe.

\section{Matériels et méthodes}

\section{Analyse immunoenzymatique}

Le dépistage ou le dosage immunologique des principales molécules responsables d'intoxications (benzodiazépines, antidépresseurs tricycliques, barbituriques, paracétamol dans le sang ; amphétamine, opiacés, cannabis, cocaïne pour les urines) est effectué par méthode immunoenzymatique EMIT sur l'automate RXL Dimension ${ }^{\circledR}$ (Dade Berhing) à l'aide des différents réactifs destinés à cet appareil. L'éthanolémie est également réalisée sur le RXL Dimension ${ }^{\circledR}$ par la méthode enzymatique proposée par Dade Berhing. Les instructions du fabriquant ont été scrupuleusement respectées pour la recherche de ces paramètres.

\section{Le Remedi ${ }^{\circledR}$}

\section{Principe}

Une recherche toxicologique plus approfondie est réalisée sur Remedi® (Rapid drug emergency identification, Biorad). Il s'agit d'un automate couplant une extraction en ligne et une séparation des molécules médicamenteuses par chromatographie liquide haute performance à une détection UV par réseau tournant balayant de 200 à $300 \mathrm{~nm}$. Le passage de l'échantillon sur différentes colonnes permet de purifier, extraire et séparer les principes actifs pouvant être contenus dans les matrices biologiques (sérum, urines, lavages gastriques). L'analyse nécessite environ une vingtaine de minutes. L'identification se fait grâce à un logiciel utilisant des algorithmes associant les données spectrales de la molécule inconnue et son temps de rétention par rapport à deux étalons internes. Les caractéristiques spectrales et chromatographiques sont comparées à celles de la bibliothèque qui regroupe environ 800 molécules et métabolites. La comparaison est illustrée par un coefficient de similitude, celui-ci devant tendre vers zéro.

L'estimation semi-quantitative de la concentration de la molécule identifiée est fonction de la hauteur du pic et d'un facteur de réponse calculé à partir de trois niveaux de concentration connue. Une estimation quantitative 
stricte nécessiterait un réajustement du facteur de réponse à chaque dosage afin de gommer toute erreur liée aux variations chromatographiques (durée de vie des colonnes, température) ce qui retarderait le délai de rendu du résultat. Cependant une estimation, même semi-quantitative, reste extrêmement précieuse dans un contexte toxicologique.

\section{Matériels et réactifs}

Tous les réactifs et consommables sont fournis par Biorad. La composition de la phase mobile et des réactifs ainsi que les conditions chromatographiques et les caractéristiques analytiques des colonnes ne sont pas communiquées par la société.

\section{Traitement des échantillons}

Cette technique ne nécessite pas de traitement préalable des échantillons hormis une filtration des sérums ; mais une hydrolyse enzymatique des urines peut être utilisée afin de libérer les opiacés des glycuroconjugués : $2 \mathrm{ml}$ d'échantillon sont traités par 1 goutte de suc d'Helix pomatia (BioSepra ${ }^{\circledR}$ SHP/HPJ) contenant 100000 UF (unités Fishmann) de beta-glucuronidase par $\mathrm{ml}$ puis placés 3 heures au bain marie à $50^{\circ} \mathrm{C}$. Le volume d'échantillon analysé est de $1 \mathrm{ml}$.

\section{Résultats}

\section{Analyse immunoenzymatique}

Les dépistages et dosages immunologiques dans le sang sont tous négatifs. L'éthanolémie est également négative.

Dans les urines, le dépistage immunologique est négatif pour la cocaïne et l'amphétamine mais positif pour les opiacés et le cannabis.

\section{Analyse chromatographique}

Le screening toxicologique par le Remedi® de l'échantillon sanguin ne met en évidence aucun toxique.

L'analyse de l'échantillon urinaire natif permet quant à elle de mettre en évidence la présence d'atropine libre à la concentration de $3,6 \mathrm{mg} / \mathrm{l}$ (coefficient de similitude : 0.008) et de scopolamine libre à $0,3 \mathrm{mg} / \mathrm{l}$ (coefficient de similitude : 0.003). Aucun opiacé n'est retrouvé (figure 1).

Après hydrolyse enzymatique des conjugués urinaires une nouvelle analyse montre la présence d'atropine totale à la concentration de $5,4 \mathrm{mg} / \mathrm{l}$, de scopolamine totale à $3 \mathrm{mg} / \mathrm{l}$ et de morphine totale en très faible quantité (figure 2).

L'analyse de la boisson verdâtre révèle également la présence de grandes quantités de scopolamine (11.7 $\mathrm{mg} / \mathrm{l}$ ) et d'atropine $(27.3 \mathrm{mg} / \mathrm{l})$ (figure 3 ).

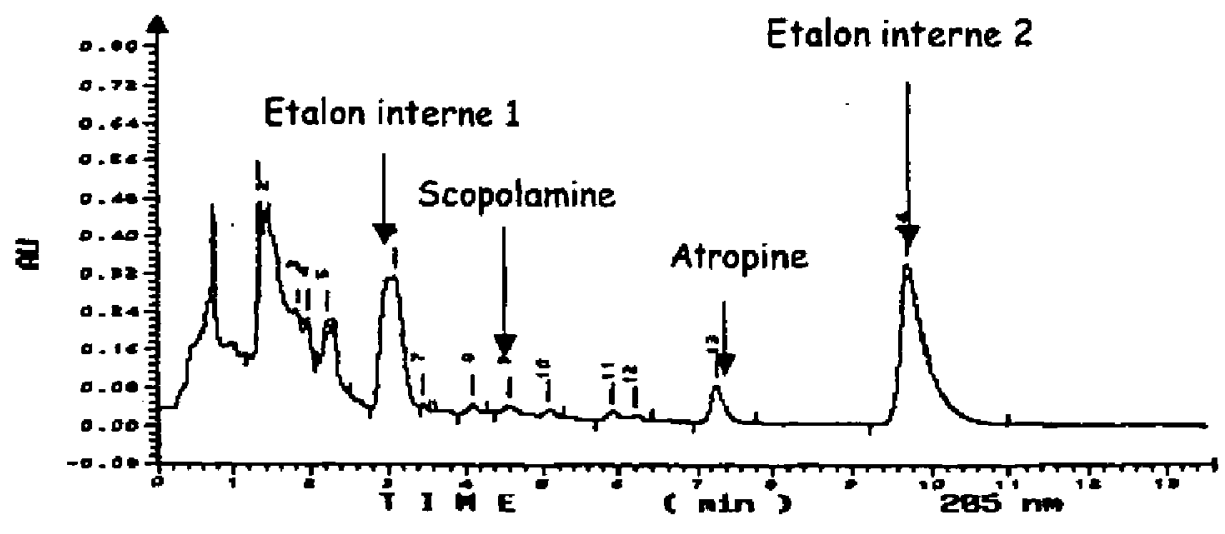

Figure 1 : Analyse chromatographique de l'échantillon urinaire sans hydrolyse.

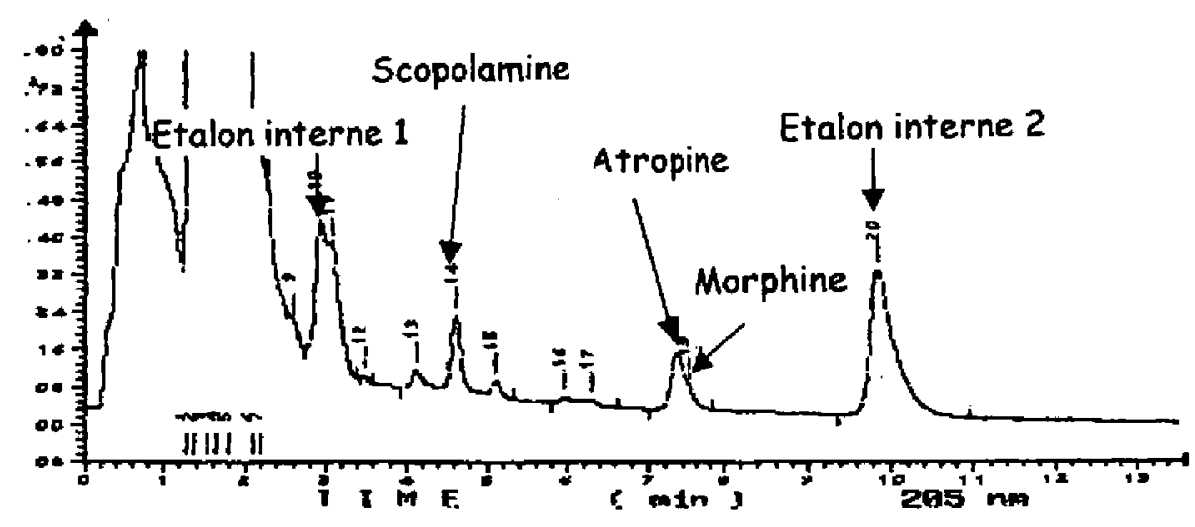

Figure 2 : Analyse chromatographique de l'échantillon urinaire après hydrolyse.

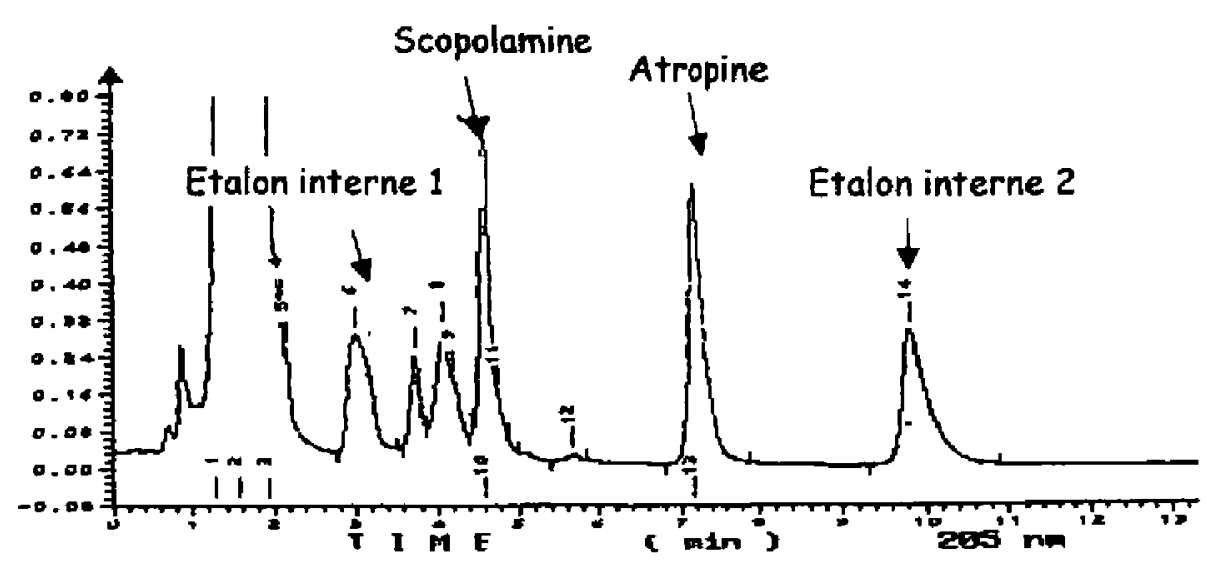

Figure 3 : Analyse chromatographique de la décoction.

\section{Discussion}

\section{Analyses}

Les différentes variétés de Datura contiennent toutes atropine, scopolamine et hyoscine avec des concentrations variant selon les espèces (2).

La mise en évidence de scopolamine et d'atropine dans la boisson et les urines du patient oriente donc vers une intoxication aux Solanacées. L'entretien ultérieur avec le psychiatre confirmera la consommation d'une tisane de Datura chez un toxicomane connu qui a tenté de substituer un manque en héroïne par le Datura. La présence en faible quantité de morphine s'explique donc par les antécédents du sujet. Aucun des alcaloïdes du Datura n'ont pu être identifiés dans le sérum du patient, 
mais il faut préciser que les limites de détection du système chromatographique sont de $0,68 \mathrm{mg} / \mathrm{l}$ pour l'atropine et $0,27 \mathrm{mg} / \mathrm{l}$ pour la scopolamine.

Sur les trois composés actifs du Datura (atropine, scopolamine, hyoscine) seule la hyoscine n'a pu être retrouvée. En effet, elle ne fait pas partie des molécules présentes dans la bibliothèque du Remedi®. Par ailleurs, il faut noter que la concentration en hyoscine est moindre dans toutes les espèces (2).

Dans un tel contexte hospitalier, pour des patients hospitalisés, le screening chromatographique Remedi® constitue un outil précieux pour l'urgence de part son utilisation simple, 24 heures sur 24 et avec un rendu de résultats rapide (environ 20 minutes). Il permet ainsi le dialogue avec le clinicien en temps réel et donc d'affirmer le diagnostic de l'intoxication au Datura.

Dans un contexte médico-légal où le caractère d'urgence n'intervient pas, la spectrométrie de masse, technique de référence, reste la méthode de confirmation à posteriori.

Outre le Remedi $($, système chromatographique entièrement automatisé dont peu de laboratoire sont équipés, différentes méthodes de dosages des alcaloïdes utilisant la chromatographie couplée à une détection UV ou à la spectrométrie de masse ont été publiées (3-6). Ces techniques utilisent en général une extraction solide-liquide $(5,6)$.

\section{Clinique et biologie}

Les signes cliniques sont d'apparition très précoce : quinze à trente minutes après l'ingestion ou l'inhalation $(7,8)$. Les alcaloïdes contenus dans le Datura sont responsables de manifestations anticholinergiques intenses caractérisées par une sécheresse des muqueuses et un tableau neurologique très polymorphe : mydriase avec paralysie de l'accommodation, somnolence, état d'excitation croissante avec agitation extrême, confusion, hallucinations visuelles et/ou auditives, désorientation pouvant aller jusqu'au délire. Une hyperthermie est souvent signalée et au cours des intoxications sévères la survenue de convulsions et de paralysies flasques a été signalée. La rétention d'urines est observée dans de rares cas.

Au niveau respiratoire et cardiovasculaire on observe une hyperventilation et une tachycardie sinusale. Dans quelques cas une hypertension a été notée.

Dans les cas graves, apparaît une dépression du système nerveux central avec détresse respiratoire évoluant vers le coma et la mort (9).

Les signes cliniques observés chez notre patient sont dans l'ensemble conformes à ceux décrits lors de ce type d'intoxication. Cependant, nous n'avons pas observé d'hyperthermie et à son arrivée au service des urgences le patient présentait une importante diminution de la pression artérielle diastolique jamais décrite dans la littérature.

Au niveau biologique la littérature décrit une augmentation des transaminases et de la lacticodéshydrogénase (7), enzymes hépatiques non dosées chez notre patient. Nous avons en revanche noté chez celui-ci une augmentation de la créatine kinase pouvant s'expliquer par son état d'agitation et la contention.

\section{Traitement et évolution}

Le traitement a été symptomatique : perfusion d'Osmotan® G5 \%, deux ampoules de Loxapac® et contention physique rendue nécessaire par l'agitation extrême du patient. L'évolution clinique a été rapidement favorable et le patient a pu quitter le service dès le lendemain. Au réveil le patient a décrit cet épisode comme particulièrement désagréable et douloureux.

Ce type d'intoxication peut constituer une véritable urgence médicale mettant en jeu le pronostic vital et nécessite donc une prise en charge dans des structures adaptées. La plupart du temps le traitement est symptomatique (surveillance des fonctions vitales, réhydratation, anxiolytique non anticholinergique). L'évolution est favorable, sans séquelles, avec une durée d'hospitalisation variant de 30 à 40 heures mais tous les sujets décrivent cette expérience comme très désagréable (9). Les graines de Datura étant très longues à digérer, il peut être intéressant de réaliser un lavage gastrique et/ou d'administrer du charbon activé. Mais ce lavage gastrique peut être difficile à réaliser compte tenu de l'état d'agitation du sujet $(9,10)$.

\section{Conclusion}

L'intoxication au Datura n'est plus exceptionnelle. La faible différence entre la dose hallucinogène et la dose toxique fait que la consommation de Datura est extrêmement dangereuse et le risque est d'autant plus grand qu'il est difficile pour le consommateur de prévoir la quantité ingérée avec ce type de préparation.

Le diagnostic de cette intoxication est clinique et souvent mal connu. Le diagnostic différentiel se discute avec d'autres étiologies du syndrome anticholinergique comme notamment les intoxications par les antidépresseurs tricycliques. L'analyse toxicologique a permis ici de confirmer l'intoxication au Datura et d'éliminer toute autre toxicomanie ou prise médicamenteuse et donc de confirmer le bien fondé du traitement. 


\section{Références}

1. Bello P., Toufik A., Gandilhon M., Giraudon I., Bonnet $\mathrm{N}$. Phénomènes émergents liées aux drogues en 2002, quatrième rapport national du dispositif TREND. Paris : OFDT, 2003.

2. Hiraoka N., Tashimo K., Kinoshita C., and Hiro'Oka M. Genotypes and alkaloid contents of Datura metel varieties. Biol. Pharm. Bull. 1996 ; 19 : 1086-9.

3. Papadoyannis I.-N., Samanidou V.-F., Theodoridis G.-A., Vasilikiotis G.-S., Kempen G.-J.-M., Beelen G.-M. A simple and quick solid phase extraction and reversed phase HPLC analysis of some tropane alkaloids in feedstuffs and biological samples. J. Liq. Chromatogr. 1993 ; $16: 975-98$.

4. Fliniaux M.-A., Manceau F., Jacquin-Dubreuil A. Simultaneous analysis of 1-hyosciamine, 1-scopolamine and dl-tropic acid in plant material by reversed phase high-performance liquid chromatography. J. Chromatogr. B. $1993 ; 644: 193-7$.

5. Namera A., Yashiki M., Hirose Y. Quantitative analysis of tropane alkaloids in biological materials by gas-chromatography-mass spectrometry. Forensic Sci. 2002 ; $130: 34-43$.
6. Oertel R., Richter K., Ebert U., Kirch W. Determination of scopolamine in human serum and microdialysis samples by liquid-tandem mass spectrometry. $\mathbf{J}$. Chromatogr. B. $2001 ; 750: 121-8$.

7. Chang S., Wu M., Deng J., Lee C., Chin T., Liao S. Poisoning by datura leaves used as edible wild vegetables. Vet. Hum. Toxicol. $1999 ; 41: 242-5$.

8. Hall R., Popkin M., McHenry L. Angel's trompet psychosis : a central nervous system anticholinergic syndrome. AM. J. Psychiatry. 1977 ; 134 : 312-4.

9. Roblot F., Montaz L., Delcoustal M., Gaboriau E., Chavagnat J., Morichaud G., Pourrat O., Scepi M., Patte D. Intoxication par Datura stramonium : le diagnostic est clinique, le traitement est symptomatique. Rev. Med. Interne. $1995 ; 16: 187-90$.

10. Salen P., Shih R., Sierzenski P., Reed J. Effect of Physostigmine and Gastric Lavage in a Datura Stramonium-Induced Anticholinergic Poisoning Epidemic. Am. J. Emerg. Med. 2003 ; 21 : 316-7. 